\title{
Article
}

\section{Impact of harness attachment point on kinetics and kinematics during sled towing.}

Bentley, Ian, Atkins, Stephen, Edmundson, Christopher James, Metcalfe, John and Sinclair, Jonathan Kenneth

Available at http://clok.uclan.ac.uk/13603/

Bentley, Ian ORCID: 0000-0002-9086-2338, Atkins, Stephen, Edmundson, Christopher James ORCID: 0000-0003-2275-7584, Metcalfe, John ORCID: 0000-0002-8414-978X and Sinclair, Jonathan Kenneth ORCID: 0000-00022231-3732 (2016) Impact of harness attachment point on kinetics and kinematics during sled towing. Journal of strength and conditioning research, 30 (3). pp. 768-776. ISSN 1064-8011

It is advisable to refer to the publisher's version if you intend to cite from the work. http://dx.doi.org/10.1519/JSC.0000000000001155

For more information about UCLan's research in this area go to http://www.uclan.ac.uk/researchgroups/ and search for < name of research Group>.

For information about Research generally at UCLan please go to http://www.uclan.ac.uk/research/

All outputs in CLoK are protected by Intellectual Property Rights law, including Copyright law. Copyright, IPR and Moral Rights for the works on this site are retained by the individual authors and/or other copyright owners. Terms and conditions for use of this material are defined in the policies page.

\section{CLoK}

Central Lancashire online Knowledge www.clok.uclan.ac.uk

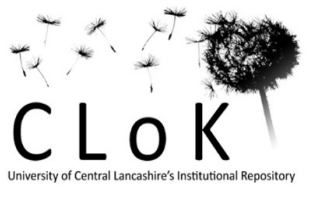




\section{Impact of harness attachment point on kinetics and kinematics during sled}

\section{towing}

Ian Bentley ${ }^{1}$, Steve Atkins ${ }^{1}$, Christopher Edmundson ${ }^{1}$, John Metcalfe ${ }^{2}$ and Jonathan

Sinclair $^{1}$

${ }^{1}$ Division of Sport, Exercise and Nutritional Sciences; and ${ }^{2}$ Division of Studies,

Management and the Outdoors, University of Central Lancashire, Preston, Lancashire

Address correspondence to lan Bentley:

School of Sport, Tourism and the Outdoors

University of Central Lancashire

Preston

PR1 2HE

Darwin Building 223

01772893511

IBentley1@uclan.ac.uk 


\section{$1 \quad$ ABSTRACT}

2 Resisted sprint training is performed in a horizontal direction, and involves similar

3 muscles, velocities and ranges of motion (ROM) to those of normal sprinting.

4 Generally, sleds are attached to the athletes via a lead $(3 \mathrm{~m})$ and harness; the most

5 common attachment points are the shoulder or waist. At present, it is not known how

6 the different harness point's impact on the kinematics and kinetics associated with

7 sled towing (ST). The aim of the current investigation was to examine the kinetics

8 and kinematics of shoulder and waist harness attachment points in relation to the acceleration phase of ST. Fourteen trained males completed normal and ST trials, loaded at $10 \%$ reduction of sprint velocity. Sagittal plane kinematics from the trunk, hip, knee and ankle were measured, together with stance phase kinetics (third footstrike). Kinetic and kinematic parameters were compared between harness attachments using one-way repeated measures analysis of variance. The results indicated that various kinetic differences were present between the normal and ST conditions. Significantly greater net horizontal mean force, net horizontal impulses, propulsive mean force and propulsive impulses were measured ( $p>0.05)$. Interestingly, the waist harness also led to greater net horizontal impulse when compared to the shoulder attachment $(p=0.000)$. In kinematic terms, ST conditions significantly increased peak flexion in hip, knee and ankle joints compared to the normal trials $(p<0.05)$. Results highlighted that the shoulder harness had a greater impact on trunk and knee joint kinematics when compared to the waist harness $(p<0.05)$. In summary, waist harnesses appear to be the most suitable attachment point for the acceleration phase of sprinting. Sled towing with these attachments resulted in fewer kinematic alterations and greater net horizontal impulse when 
compared to the shoulder harness. Future research is necessary, in order to explore the long-term adaptations of these acute changes.

Keywords: acceleration, biomechanics, resisted sprint training

Word count:

30

\section{INTRODUCTION}

Sprinting is essential for success in many sports $(11,12,13,27)$. In field sports

where the need to reach the ball first, or be in position for a play to develop is decisive, speed is a crucial factor $(22,29)$. Sprint velocity is a product of stride length and stride frequency. To increase velocity, one or both of these components must be increased $(22,33)$. Stride length and stride frequency can be increased by exerting larger forces or increasing the rate of force development (RFD) during the stance phase $(15,24,35)$. It is generally accepted that while maximum velocity is important in field sports, the ability to accelerate is seen as being of greater significance (10, 27).

The kinematic and kinetic characteristics of the acceleration and maximal velocity phases of sprinting are quite different. The acceleration phase requires a greater forward trunk lean (16). Kugler et al. (20) proposed that if the force vector points further forward (trunk lean) then the ratio of vertical to propulsive force will be biased towards forwards propulsion. In this instance, greater ground reaction force (GRF) can be applied without the negative effects associated with high vertical force application, such as short contact times. In contrast, at maximum velocity, athletes must preserve optimal postural stability, minimising braking and increasing vertical 
forces. Greater vertical ground reaction forces are essential in allowing faster sprinters to reduce foot contact time during the stance phase (36).

The development of various resisted sprint training modalities, such as sled, parachute, and bungees, are providing coaches with alternative or additional sport specific training strategies to more traditional methods. During ST, the external resistance is provided by the mass of the sled and the coefficient of friction between the sled and the surface (8). Resisted sprint training is performed in a horizontal direction, and involves the relevant muscles, velocities and ranges of motion similar to those of normal sprinting $(1,19)$.

Sled loading strategies, as well as the sets and repetitions used to implement ST, remain equivocal $(1,9,23,26,28)$. There are several different methods by which sleds can be loaded; sled loading based on an absolute load or relative load relating to body mass have been commonly employed, however these methods do not take the athlete's strength capabilities into consideration (14, 34). As such, loading sleds based on a reduction of sprint velocity is the preferred method $(2,7,25,34)$. Previous investigations have implemented various sled loadings ranging from a $5 \mathrm{~kg}$ absolute load to $32.2 \%$ body mass $(23,37)$. Many researchers have found lighter sled loads to be the most effective as they have been shown to have less impact on contact time variables, joint angles and $\operatorname{ROM}(17,26,28)$. Several researchers have used sled loadings based on a $10 \%$ decrement in sprint velocity to improve peformance $(7,25,33)$. Whilst information on loading strategies is undergoing a process of confirmation, there is a dearth of literature relating to the practicalities of ST, notably with regard to attachments for harness systems. 
Lawrence et al. (21) investigated the effects of different harness attachment points (shoulder and waist) on walking sled pulls. They reported differences in joint moments between the different attachments, concluding that the shoulder harness would challenge the knee extensors, and the waist harness the hip extensors. Over time, it is expected that the different harness attachments would lead to positive strength adaptations related to the aforementioned joints, thereby allowing coaches to tailor the sled pulls specifically to areas of weakness.

83

Generally, sleds are attached to the athletes via a lead $(3 \mathrm{~m})$ and harness system, the most common being a shoulder or waist attachment point, At present, it is not known how the different harness attachment points impact on ST kinematics and kinetics. Therefore, the purpose of this study was to investigate the sprint kinematics and kinetics of ST during the acceleration phase when sleds were loaded to cause a $10 \%$ reduction in sprint velocity. Subjects completed sprint trials under different conditions (normal sprinting, shoulder attachment and waist attachment). It was hypothesised that 1) differences between the kinetic parameters would be negligible between conditions, 2) both sled trials would be significantly different from the normal sprint condition in terms of lower limb and trunk kinematics, and 3) the attachment point would impact trunk, hip, knee and hip joint kinematics differently. The findings will allow coaches to alter their use of ST to better suit the acceleration phase.

\section{METHODS}

\section{Experimental Approach to the Problem}


100 This study used a cross-over design to compare the effects of different harness attachments during ST. Fourteen resistance trained males performed a series of $6 \mathrm{~m}$ sprints in three different conditions (normal, with shoulder and waist attachments). The key dependant variables were the sagittal plane kinematic measures of the lower extremities and trunk, the kinetic data obtained from the force platform and various contact time measures.

\section{Subjects}

Fourteen resistance trained males (age: $26.7 \pm 3.5$ years; mass: $84.2 \pm 12.3 \mathrm{~kg}$; stature: $174.4 \pm 6.4 \mathrm{~cm}$ ) participated in this study. All subjects were resistance trained (2 years minimum) with ST experience. The sample size was calculated based on previous acute ST investigations $(14,21)$. All subjects gave written and informed consent before attending the testing sessions. The project was reviewed and approved by the institutional ethics committee of the University of Central Lancashire, in accordance with the principles of the Declaration of Helsinki. No external funding was provided by any of the harness or sled manufacturers used in this study.

\section{Procedures}

One week prior to testing, all subjects completed a familiarization session. During this session subjects were able to practice ST using the different harness attachment points. The same sled was used during all of the loaded trials. The sled was attached to the subjects using a $3 \mathrm{~m}$ non-elasticated attachment cord, and either a double shoulder strap or single waist belt. Using a $6 \mathrm{~m}$ sprint as a baseline, sleds were loaded so that sprint velocity was reduced by $10 \%$ (waist condition), as 
recommended by Kawamori et al. (17). Sprint velocity was monitored using infrared timing lights (Smartspeed Ltd., United Kingdom).

Targeting occurs when participants deliberately lengthen or shorten the stride prior to force plate contact (32). These stride alterations have been shown to significantly impact on sagittal plane joint kinematics (6). Research shows that participants are able to run across an embedded force plate without significantly adjusting their stride mechanics (32). No studies have looked at how sprinting over an embedded force plate impacts on lower body kinematics. However, in the current study measures were taken to ensure that no force plate targeting took place. Firstly, the familiarization session was used to determine an individual starting position for each subject. Starting positions were adjusted so that each participant's right foot contacted the force plate on their third step. Starting positions of the ST trials were also adjusted accordingly and practiced until participants consistently landed on the force plate. In order to standardise starting positions, trials began in a 3 point position. Each participant chose to start with his left foot leading in the 3 point starting position. Regardless of the starting point, subjects sprinted a total distance of $6 \mathrm{~m}$.

Subjects were asked not to participate in any physical activity 24 hours before the testing session. No food was allowed to be consumed during testing, though water was allowed. The testing session began with a standardised warm-up consisting of

147 jogging (5 minutes), dynamic stretching (5 minutes) and a number of sprints building up to maximum intensity ( $2 \times 75 \%, 2 \times 90 \%$ and $2 \times$ maximum). 
150 Previous research has shown that ST trials can impact on the kinematics of any subsequent normal sprint trials (17). Thus, the normal sprint trials were completed before either of the sled conditions (shoulder or waist). Once the normal sprint trials had been recorded, the ST trials were randomised. Testing procedures were identical to those described previously in the familiarisation section. All subjects had 2 minutes recovery between each of the sprint trials. Five trials were collected for each of the conditions. Again, subjects sprinted a distance of $6 \mathrm{~m}$ in a $22 \mathrm{~m}$ lab. An embedded force platform, sampling at $1000 \mathrm{~Hz}$, was positioned at approximately $3 \mathrm{~m}$ from the start (model 9281CA; dimensions $=0.6 \times 0.4 \mathrm{~m}$, Kistler Instruments Ltd). In order for the trials to be deemed successful, the whole foot had to contact the force platform. Trials were discarded in cases where any part of the foot did not land the force platform. Sprint times were generated for every trial, and any trials in which sprint velocity deviated more than $\pm 5 \%$ of the initial trial in that condition were not used in the final analysis. In this instance, an extended recovery period of 4 minutes was implemented and trails were repeated. malleolus, lateral malleolus, medial epicondyle, lateral epicondyle, acromion process (both), T12 and C7 (4). The pelvis segment was defined, using additional markers on the anterior (ASIS) and posterior (PSIS) superior iliac spines. Hip joint centre was determined based on the Bell et al., (3) equations via the positions of the PSIS and 
175 ASIS markers. During dynamic trials the foot segment was tracked using the 176 calcaneus, $1^{\text {st }}$ and $5^{\text {th }}$ metatarsal heads. Rigid cluster tracking markers were also 177 positioned on the right shank and thigh segments (5). The ASIS, PSIS and greater trochanters were used as tracking markers for the pelvis. The trunk was tracked using markers at both acromion processes, as well as the T12 marker. A static calibration was completed and used as reference for anatomical marker placement in relation to the tracking markers, after which all non-tracking markers were removed.

Motion files were exported as C3D files and quantified using Visual 3-D (C-Motion Inc., Germantown, USA) and filtered at $12 \mathrm{~Hz}$ using a Butterworth $4^{\text {th }}$ order filter. Three dimensional kinematics of the lower extremities and trunk were calculated using an $X Y Z$ cardan sequence of rotations ( $X$ represents the sagittal plane, $Y$ represents the coronal plane and $Z$ the transverse plane). The relevant segments (thorax, thigh, shank and virtual foot) and reference segments (pelvis, thigh and shank) were used to calculate joint angles of the trunk, hip, knee and ankle joints respectively. All kinematic waveforms were normalised to $100 \%$ of the stance phase and then processed trials were averaged. Various kinematic measures from the trunk, hip, knee and ankle joints were investigated: angle at foot-strike, angle at toeoff, peak angle, ROM from foot-strike to toe-off, and the relative ROM (the angular displacement from foot-strike to peak angle). Resultant velocity at toe-off was calculated using the vertical and horizontal centre of mass. These variables were extracted from each of the 5 trials for each joint, data was then averaged within subjects for a comparative statistical analysis. 
200 Contact time was determined as time over which $20 \mathrm{~N}$ or greater of vertical force was

201

202

203

204

205

206

207

208

209

210

211

212 applied to the force platform (30). The durations of the braking and propulsive phases were based on anterior and posterior horizontal GRF. Peak GRF was determined for the following components: vertical, braking, propulsive. Vertical impulse was calculated as the area under the vertical ground reaction force-time curve minus body weight impulse over the time of ground contact. The braking and propulsive impulses were determined by integrating all the negative and positive values of horizontal GRF, respectively, over the time of ground contact (17). Net horizontal impulse was calculated as propulsive impulse minus the absolute value of braking impulse. Similarly, mean values of vertical and net horizontal GRF were obtained by dividing respective impulse values by the contact time, whereas mean braking and propulsive GRF were calculated by the time duration of braking and propulsive phases, respectively (17). All GRF measures were expressed relative to total body mass.

\section{Statistical Analysis}

Descriptive statistics were calculated and presented as mean \pm SD. One-way within subjects analysis of variance (ANOVA) was used to compare the means of the different conditions (normal, waist and shoulder) with the different outcome measures (velocity, contact time, kinematics, kinetics). The significance level was set at $p \leq 0.05$. Post hoc pairwise comparisons were conducted on all significant main effects using a Bonferroni adjustment to control for type I error. Effect sizes were calculated using partial $\mathrm{Eta}^{2}\left(\mathrm{p \eta}^{2}\right)$. All statistical analyses were undertaken using SPSS (Version 22, IBM SPSS Inc., Chicago, USA). 


\section{RESULTS}

226 Table 1 presents the stance phase velocity and contact time data. The kinetic

227 measures are presented in Table 2. Tables 3-6 present the sagittal plane kinematic parameters from the trunk, hip, knee and ankle joints. Figure 1 presents the mean sagittal plane angular kinematics during the stance phase.

The mean sagittal kinematic waveforms were qualitatively similar (Figure 1), although statistical differences were observed at the trunk, hip, knee and ankle joints (Tables 3-6).

@@@Figure 1 inserted near here@@@

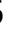

The results indicate that a significant main effect was observed for sprint velocity $\left(p<0.01, p \eta^{2}=0.87\right)$. Post hoc analysis revealed that sprint velocity was significantly reduced during the waist $(p=0.000)$ and shoulder $(p=0.000)$ trials compared to the normal trials. There was no significant difference between the ST conditions $(p=$ $0.616)$

Similarly, a significant main effect was observed for the contact time of the stance leg $\left(p<0.01, p \eta^{2}=0.66\right)$. Post hoc analysis revealed that contact times of the stance leg were significantly shorter in the normal condition compared to the waist $(p=$ $0.000)$ and shoulder $(p=0.000)$ attachments. There was no significant difference between ST conditions ( $p=0.073$ ). Results highlighted a significant main effect for 
248 the duration of the propulsive phase of the stance $\left(p<0.01, p \eta^{2}=0.48\right)$. Post hoc

249 tests indicated that the propulsive phase was significantly longer during the waist ( $p$ $250=0.024)$ and shoulder $(p=0.002)$ attachment trials compared to the normal sprint 251 trials. There was no significant difference between ST conditions $(p=0.841)$.

The results (Table 2) show that there was a significant main effect for net horizontal mean force $\left(p<0.001, p \eta^{2}=0.547\right)$. Post hoc tests revealed that the normal condition resulted in significantly lower net horizontal mean force than the shoulder attachment $(p=0.020)$ and the waist condition $(p=0.001)$. There was no significant difference between the ST conditions $(p=0.056)$. Similarly, there was a significant main effect for the net horizontal impulse between conditions $\left(p<0.001, \mathrm{p \eta}^{2}=0.742\right)$. Post hoc tests indicated that both ST conditions were significantly greater than the normal sprint trials $(p=0.000)$. The net horizontal impulses produced during the waist attachment condition were significantly larger than the shoulder condition $(p=$ 0.045). There was a significant main effect for the propulsive mean force $(p<0.05$, $p \eta^{2}=0.329$ ). Post hoc tests revealed that the waist condition led to significantly greater mean propulsive GRF than the normal condition $(p=0.004)$. There was no significant difference between the ST conditions $(p=0.056)$. Finally, a significant main effect was observed for propulsive impulse measures $\left(p<0.001, p \eta^{2}=0.746\right)$. Post hoc tests revealed that the normal condition resulted in significantly lower propulsive impulse measures than the shoulder attachment $(p=0.000)$ and the waist 
271 condition $(p=0.000)$. There was no significant difference between the ST conditions $272 \quad(p=0.063)$.

276 The results (Table 3 ) show that in the sagittal plane there was a significant main 277 effect for the magnitude of ROM for the trunk $\left(p<0.001, p \eta^{2}=0.493\right)$. Post hoc tests revealed that trunk ROM was significantly lower during the shoulder condition compared to the normal $(p=0.000)$ and waist $(p=0.000)$ conditions. A significant main effect was observed for the relative $R O M$ of the trunk $\left(p>0.001, p \eta^{2}=0.410\right)$. Post hoc tests indicated that relative trunk ROM was significantly greater in the shoulder condition compared to the normal sprinting condition $(p=0.001)$.

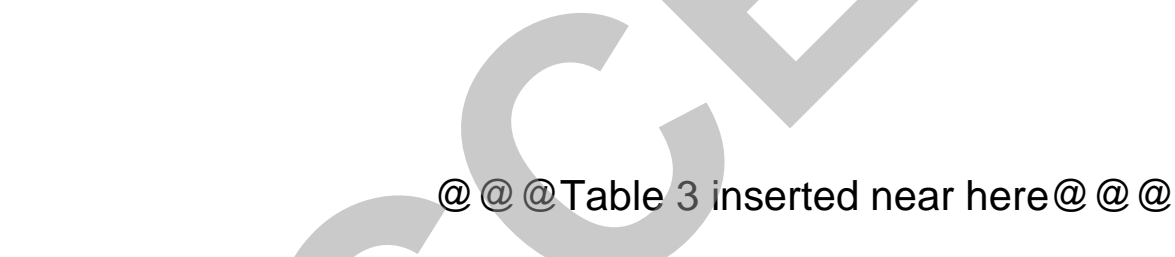

The results (Table 4) show that in the sagittal plane there was a significant main effect for hip joint angle at foot-strike $\left(p<0.001, p^{2}=0.47\right)$. Flexion at the hip joint was significantly greater at foot-strike during the waist $(p=0.015)$ and shoulder $(p=$ 0.004) attachment trials compared to the normal trials. There was no significant difference between the ST trials $(p=1.000)$. Similarly, the results indicate that there was a main effect for hip joint angle at toe-off $\left(p<0.05, p \eta^{2}=0.38\right)$. Extension was greater in the normal trials compared to the waist $(p=0.015)$ and shoulder $(p=$ 
0.035) attachment trials. There was no significant difference between ST trials $(p=$

294 1.000). Finally, a significant main effect was found for peak hip flexion $\left(p<0.001, p \eta^{2}\right.$ $295=0.47)$. The peak hip joint angle was significantly lower in the normal sprint trials

296

297

298

299

300

301

302

303

304

305

306

307

308

309

310

311

312

313

314

315

316 compared to the waist $(p=0.015)$ and shoulder $(p=0.004)$ attachment conditions. There was no significant difference between the ST sled trials $(p=1.000)$.

@@@Table4 inserted near here@@@

The results (Table 5) show that in the sagittal plane there was a significant main effect for knee joint angle at foot-strike $\left(p<0.001, \mathrm{p \eta}^{2}=0.73\right)$. Post hoc tests revealed that knee joint flexion was significantly greater at foot-strike during the waist $(p=0.000)$ and shoulder $(p=0.000)$ attachment sled trials compared to the normal sprint trials. There was no significant difference between ST conditions $(p=0.441)$. The results indicate that there was a significant main effect for knee joint angle at toe-off $\left(p<0.05, p \eta^{2}=0.36\right)$. Knee joint extension was greater in the normal trials compared to the waist $(p=0.018)$ and shoulder $(p=0.016)$ attachment trials. There was no significant difference between ST trials $(p=1.000)$. A significant main effect was found for peak knee joint angle $\left(\mathrm{p}<0.001, \mathrm{p \eta}^{2}=0.73\right)$. Post hoc analysis revealed that all of the conditions were significantly different from one another. Knee flexion in the normal trials was lower than the waist $(p=0.001)$ and shoulder $(p=$ 0.000) attachment trials. Knee flexion was significantly greater in the shoulder attachment condition compared to the waist attachment trials $(p=0.037)$. Finally, there was a significant main effect for the magnitude of ROM at the knee joint $\left(p<0.05, p^{2}=0.29\right)$. Post hoc tests indicated that knee joint ROM was significantly 
smaller in the normal condition compared to the shoulder attachment condition $(p=$ 0.036). There was no significant difference between the normal and waist attachment trials $(p=0.461)$.

The results (Table 6 ) show that in the sagittal plane there was a significant main effect for ankle joint angle at foot-strike $\left(p<0.001, p \eta^{2}=0.4\right)$. Post hoc tests indicated that dorsi-flexion was significantly greater at foot-strike during the waist $(p=0.041)$ and shoulder $(p=0.006)$ attachment trials compared to the normal sprint trials. There was no significant difference between the ST conditions $(p=0.494)$. Finally, a significant main effect was found for peak ankle dorsi-flexion $\left(p<0.001, p \eta^{2}=0.46\right)$. Peak ankle dorsi-flexion was significantly lower in the normal trials compared to the waist $(p=0.034)$ and shoulder $(p=0.002)$ attachment conditions. There was no significant difference between the ST trials $(p=0.248)$.

@@@Table 6 inserted near here@@@

\section{DISCUSSION}

The aim of this investigation was to examine the kinematics and kinetics of ST when different harness attachment points were used (shoulder and waist). Sleds were loaded to cause a $10 \%$ reduction in sprint velocity over a $6 \mathrm{~m}$ distance. To the authors knowledge this is the first study to use a motion capture system to measure 
340 the sagittal plane kinematics of ST. This study will have practical implications to strength and conditioning coaches looking to improve acceleration performance.

Results show that there were significant kinetic differences between the ST conditions and the normal sprint trials, supporting the rejection of the first hypothesis. These findings are contradictory to those of Kawamori et al. (17) who measured various GRF variables with a similar $10 \%$ BM sled loading. Both ST conditions were significantly different from the normal condition in numerous parameters: net horizontal mean force, net horizontal impulse, and propulsive impulse. Again, in contrast to Kawamori et al. (17) the ST conditions in this study resulted in longer ground contact times and propulsive phase contact times compared to the normal sprint trials. The increased propulsive contact times were not surprising as more propulsive force was required to overcome the extra resistance provided by the ST. However, the increased net horizontal force and propulsive impulse measures could be explained by longer ground contact times thus allowing more time to push in a horizontal direction.

Previous studies have reported that a $10 \%$ sled loading (BM or velocity reduction) had no significant acute impact on sprint kinematics $(27,28)$. However, we hypothesised that sprint kinematics during ST would be different from the normal sprint condition. The results of the present study supported our hypothesis. There were significant differences between normal sprint trials and both ST conditions in the sagittal plane at the hip, knee and ankle joints. Peak hip flexion, flexion at footstrike, and flexion at toe-off were greater during the ST trials. Similarly knee joint flexion was significantly greater for the ST conditions. Dorsi-flexion was significantly 
greater in the ST conditions at foot-strike as were the peak angles recorded. These findings contradict the theory that the $10 \%$ loading is the ideal because kinematics are not significantly altered $(26,28)$. It is beyond the scope of the present study to suggest what the longer-term implications of these alterations might be.

370 Finally, the third hypothesis was also accepted. Both harness attachment points altered kinematics differently. During ST, the harness attachment points affected the athletes differently to those reported previously in heavy walking sled pulls (21). Trunk ROM was significantly lower during the shoulder attachment condition compared to the other conditions (Table 3). In contrast, trunk relative ROM was only significantly greater in the shoulder condition compared to the normal trials. The shoulder attachment lead to significantly greater peak knee flexion when compared to the waist harness (Table 5). The knee joint ROM in the shoulder condition was significantly greater than the normal condition, whereas differences between the waist condition and the other conditions were negligible (Table 5).

Unexpectedly, the ST harness attachment points also impacted stance phase kinetics differently. The waist harness led to significantly greater net horizontal impulse compared to the shoulder attachment condition. Furthermore, the waist condition resulted in significantly greater propulsive mean GRF when compared to the normal sprint condition. Importantly, none of the ST contact time measures were significantly different. Previous researchers (18) have highlighted net horizontal impulses and propulsive force as being key to achieving high acceleration, as such it would appear that the waist harness is more suitable when training for the acceleration phase of sprinting. It seems apparent that the kinematic alterations 
caused by the waist harness made the line of action more horizontal, resulting in greater net horizontal impulse.

Our results highlighted differences in trunk angle between ST conditions. Previous investigations have also discussed the importance of trunk lean during ST. Alcaraz et al. (1) suggested that shoulder attachments would increase trunk lean to a greater extent than a waist harness attachment point. They reported, that due to the applied load being higher than the hips (pivot point), the athletes would have to compensate and increase trunk lean. It was proposed that the greater trunk lean would impact on the athletes force vector so that more propulsive GRF was applied compared to vertical GRF. Conversely, when sleds were attached via waist belts the load passed through the hips, as such these attachments did not promote an increased trunk lean (1). As such, the authors suggested that shoulder harness attachments would be more beneficial when training for the acceleration phase, and waist attachments could be more suited to the maximum velocity phase (1). In contrast, results from this study indicated that negligible differences in peak flexion, angle at foot-strike and toe-off between exist between ST conditions at the trunk. The only differences were that trunk ROM was significantly lower during the shoulder attachment condition when compared to the other conditions. Interestingly, the trunk relative ROM was only significantly greater in the shoulder condition compared to the normal trials.

410 Importantly, kinematic differences between the waist and normal sprint conditions were negligible. Therefore, our findings suggest that when the ST harness attachment is further away from the hips it alters trunk kinematics to a greater extent, thus reducing net horizontal impulse. 
415 The all-male resistance trained testing population is a limitation. Previous 416 investigations have demonstrated that females exhibit distinct lower body kinematics 417 when compared with males (31). As such, the results are limited to this population 418 and may not be applicable to female athletes. Additionally, this study only looked at 419 the harness attachment implications at a set sled loading (10\% reduction in sprint 420 velocity). Numerous investigations have highlighted that the kinetic and kinematic alterations differ greatly dependant on sled loading $(9,17,26,28)$. Thus, the findings from the present study will not be transferable to different sled loading strategies or 423 the other phases of sprinting.

\section{PRACTICAL APPLICATIONS}

426 The current investigation provides new information regarding the influence of different harness attachment configurations on the kinetics and kinematics of ST. The results indicate that ST, with the commonly prescribed loading to cause a $10 \%$ decrement in sprint velocity, will alter kinematics at the trunk, hip, knee, and ankle joints. Similarly, both ST conditions led to significant GRF alterations when compared to normal sprinting. The kinematic and kinetic alterations observed in this study differ between the waist and shoulder attachment points. Our results suggest that the waist attachment point appears to be the most suitable when training for the acceleration phase of sprinting. Sled towing with this attachment led to fewer kinematic alterations and greater net horizontal impulses when compared to the shoulder attachment trials. Future research is necessary to explore how the observed harness attachment alterations impact on sprint performance/kinematics/kinetics after prolonged ST training interventions. 
1. Alcaraz, PE, Palao, M, Elvira, JLL, and Linthorne, NP. Effects of three types of resisted sprint training devices on the kinematics of sprinting at maximal velocity. J Strength Cond Res, 22 (2): 1-8, 2008.

2. Alcaraz, PE, Elvira, JLL, and Palao, JM. Kinematic, strength, and stiffness adaptations after a short-term sled towing training in athletes. Scandinavian J Med Sci Sports, 10.111: 1600-0838, 2012.

3. Bell, AL, Brand, RA, Pedersen, DR. Prediction of hip joint centre location from external landmarks. Hum Mov Sci, 8: 3-16, 1989.

4. Cappozzo, A, Catani, F, Leardini, A, Benedeti, MG, and Della, CU. Position and orientation in space of bones during movement: Anatomical frame definition and determination. Clin Biomech, 10: 171-178, 1995.

5. Cappozzo, A, Cappello, A, Croce, U, Pensalfini, F. Surface-marker cluster design criteria for 3-D bone movement reconstruction. IEEE Transactions on Biomed Eng, 44: 1165-1174, 1997.

6. Challis, J.H. The variability in running gait caused by force plate targeting. J Appl Biomech, 17: 77-83, 2001.

7. Clark, KP, Stearne, DJ, Walts, CT, and Miller, AD. The longitudinal effects of resisted sprint training using weighted sleds vs. weighted vests. J Strength Cond Res, 24 (12): 3287-3295, 2010.

8. Cronin, J, and Hansen, KT. Resisted sprint training for the acceleration phase of sprinting. Strength Con J, 28 (4): 42-51, 2006.

9. Cronin, J, Hansen, K, Kawamori, N, and McNair, P. Effects of weighted vests and sled towing on sprint kinematics. Sports Biomech, 7 (2): 160-172, 2008. 
10.Dawson, B, Hopkinson, R, Appleby, B, Stewart, G, and Roberts, C. Player movement patterns and game activities in the Australian Football League. $\mathrm{J}$ Sci Med Sport, 7: 278-291, 2004.

11. Duthie, GM, Pyne, DB, Marsh, DJ, and Hooper, SL. Sprint patterns in rugby union players during competition. J Strength Cond Res, 20 (1): 208-214, 2006.

12. Frost, DM, Cronin, JB, and Levin, G. Stepping backward can improve sprint performance over short distances. J Strength Cond Res, 22 (3): 918-922, 2008.

13. Harrison, AJ. Biomechanical factors in sprint training: where science meets coaching. Int Symp Biomech Sports, 28: 36-41, 2010.

14. Harrison, AJ, and Bourke, G. The effect of resisted sprint training on speed and strength performance in male rugby players. J Strength Cond Res, 23 (1): 275-283, 2009.

15. Hunter, JP, Marshall, RN, and McNair, PJ. Interaction of step length and step rate during sprint running. Med Sci Sport Ex, 36.2: 261-271, 2004.

16. Hunter, JP, Marshall, RN, and McNair, PJ. Relationships between ground reaction force impulse and kinematics of sprint-running acceleration. Journal of Applied Biomechanics, 21: 31-43. 2005.

17. Kawamori, N, Newton, R, and Nosaka, K. Effects of weighted sled towing on ground reaction force during the acceleration phase of sprint running. J Sports Sci, 1-7, 2014.

18. Kawamori, N, Nosaka, K, and Newton, R. Relationships between ground reation impulse and sprint acceleration performance in team sport athletes. $\mathrm{J}$ Strength Cond Res, 27 (3): 568-573, 2013. 
19. Kristensen, GO, Tillaar, R, and Ettema, GJ. Velocity specificity in early-phase sprint training. J Strength Cond Res, 20 (4): 833-837, 2006.

20. Kugler, F, and Janshen, L. Body position determines propulsive forces in accelerated running. Journal of Biomechanics, 43, 343-348. 2010. 
28. Murray, A, Aitchison, TC, Ross, G, Sutherland, K, Watt, I, McLean, D, and Grant, S. The effect of towing a range of relative resistances on sprint performance. J Sports Sci, 23 (9): 927-935, 2005.

29. Silvestre, R, West, C, Maresh, CM, and Kraemer, WJ. Body composition and physical performance in men's soccer: A study of a National Collegiate Athletic Association Division 1 team. J Strength Cond Res, 20 (1): 177-183, 2006.

30. Sinclair, J, Edmundson, CJ, Brooks, D, and Hobbs, SJ. Evaluation of kinematic methods of identifying gait events during running. Int $J$ Sports Sci Eng, 5: 188-192, 2011.

31. Sinclair, J, Greenhalgh, A, Edmundson, CJ, Brooks, D, and Hobbs, SJ. Gender differences in the kinetics and kinematics of distance running: Implications for footwear design. Int J Sports Sci Eng, 6: 118-128, 2012.

32. Sinclair, J, Hobbs, SJ, Taylor, PJ, Currigan, G, and Greenhalgh, A. The influence of different force and pressure measuring transducers on lower extremity kinematics measured during running. J Appl Biomech, 30: 166-172, 2014.

33.Spinks, CD, Murphy, AJ, Spinks, WL, and Lockie, RG. The effects of resisted sprint training on acceleration performance and kinematics in soccer, rugby union, and Australian football players. J Strength Cond Res, 21 (1): 77-85, 2007.

34. West, DJ, Cunningham, DJ, Bracken, RM, Bevan, HR, Crewther, BT, Cook, CJ, and Kilduff, LP. Effects of resisted sprint training on acceleration in professional rugby union players. J Strength Cond Res, 27 (4): 1014-1018, 2013. 
35. Weyand, PG, Sandell, RF, Prime, DNL, and Bundle, MW. The biological limits to running speed are imposed from the ground up. J Appl Phys, 108: 950-961, 2010.

36. Weyand, PG, Sternlight, DB, Bellizzi, MJ, and Wright, S. Faster top running speeds are achieved with greater ground forces not more rapid leg movements. Journal of Applied Physiology, 89, 1991-1999. 2000.

37.Zafeiridis, A, Saraslanidis, P, Manou, V, loakimidis, P, Dipla, K, and Kellis, S. The effects of resisted sled-pulling sprint training on acceleration and maximum speed performance. The Journal of Sports Medicine and Physical Fitness, 45 (3): 284-290. 2005.

\section{Figure labels}

Figure 1. Mean trunk (a) hip (b) knee (c) and ankle (d) joint angles in the sagittal plane for the normal (bold line), shoulder (dashed line) and waist (dotted line) conditions. 
Table 1. Velocity and contact variables (means and standard deviations) under the different conditions (normal, shoulder and waist).

\begin{tabular}{llll}
\hline & Normal & Shoulder & Waist \\
\hline Velocity $\left({\left.\mathrm{m} . \mathrm{s}^{-1}\right)}\right.$ & $5.61 \pm 0.34$ & $5.08 \pm 0.3^{*}$ & $5.13 \pm 0.31^{*}$ \\
Contact time (s) & $0.17 \pm 0.02$ & $0.19 \pm 0.03^{*}$ & $0.19 \pm 0.22^{*}$ \\
Braking phase duration (s) & $0.02 \pm 0.02$ & $0.02 \pm 0.01$ & $0.01 \pm 0.00$ \\
Propulsive phase duration (s) & $0.15 \pm 0.02$ & $0.18 \pm 0.02^{*}$ & $0.17 \pm 0.02^{*}$ \\
& & & \\
\hline
\end{tabular}

* Significantly different from normal sprinting $\mathrm{p} \leq 0.05$ 
Table 2. Kinetic variables (means and standard deviations) from the third step under the different conditions (normal, shoulder and waist).

\begin{tabular}{|c|c|c|c|}
\hline & Normal & Shoulder & Waist \\
\hline Vertical peak force $\left(\mathrm{N} \cdot \mathrm{kg}^{-1}\right)$ & $10.28 \pm 2.11$ & $9.56 \pm 2.07$ & $9.77 \pm 1.73$ \\
\hline Vertical mean force $\left(\mathrm{N} \cdot \mathrm{kg}^{-1}\right)$ & $3.58 \pm 1.20$ & $3.14 \pm 1.00$ & \\
\hline Vertical impulse $\left(\mathrm{m}^{\prime} \mathrm{s}^{-1}\right)$ & $0.61 \pm 0.16$ & $0.60 \pm 0.18$ & \\
\hline Net horizontal mean force $\left(\mathrm{N} \cdot \mathrm{kg}^{-1}\right)$ & $3.23 \pm 0.58$ & & \\
\hline Net horizontal impulse $\left(\mathrm{m} \cdot \mathrm{s}^{-1}\right)$ & $0.55 \pm 0.08$ & $.67 \pm 0.08 *+$ & $0.71 \pm 0.10^{*}$ \\
\hline Braking peak force $\left(\mathrm{N} \cdot \mathrm{kg}^{-1}\right)$ & & & $2.86 \pm 1.64$ \\
\hline Braking mean force $\left(\mathrm{N} \cdot \mathrm{kg}^{-1}\right)$ & & $1.48 \pm 0.94$ & $1.28 \pm 0.91$ \\
\hline Braking impulse $\left(\mathrm{m} \cdot \mathrm{s}^{-1}\right)$ & $0.03 \pm 0.01$ & $0.03 \pm 0.01$ & $0.02 \pm 0.01$ \\
\hline Propulsive peak force $\left(\mathrm{N} \cdot \mathrm{kg}^{-1}\right)$ & $6.90 \pm 0.76$ & $6.99 \pm 0.81$ & $7.16 \pm 0.70$ \\
\hline Propulsive mean force $\left(\mathrm{N} \cdot \mathrm{kg}^{-1}\right)$ & $3.81 \pm 0.60$ & $4.00 \pm 0.54$ & $4.26 \pm 0.53^{*}$ \\
\hline Propulsive impulse $\left(\mathrm{m}^{\prime} \mathrm{s}^{-1}\right)$ & $0.58 \pm 0.08$ & $0.70 \pm 0.07 *$ & $0.73 \pm 0.09 *$ \\
\hline
\end{tabular}

* Significantly different from normal sprinting $p \leq 0.05$

+ Significantly different from waist attachment condition $p \leq 0.05$ 
Table 3. Trunk kinematics (means and standard deviations) under the different conditions (normal, shoulder and waist).

\begin{tabular}{llll}
\hline $\begin{array}{l}\text { X (+=flexion/- } \\
\text { =extension) }\end{array}$ & Normal & Shoulder & Waist \\
& & & \\
\hline Angle at foot-strike $\left(^{\circ}\right)$ & $7.62 \pm 9.42$ & $6.75 \pm 10.19$ & $8.63 \pm 10.10$ \\
Angle at toe-off $\left(^{\circ}\right)$ & $-1.83 \pm 8.70$ & $1.89 \pm 10.56$ & $1.21 \pm 10.71$ \\
& & & $11.96 \pm 11.67$ \\
Peak flexion $\left({ }^{\circ}\right)$ & $9.42 \pm 10.03$ & $11.27 \pm 10.45$ & $8.73 \pm 3.86$ \\
Range of movement $\left(^{\circ}\right)$ & $9.46 \pm 3.71$ & $4.86 \pm 3.90^{*}+$ & $3.33 \pm 3.56$ \\
$\begin{array}{l}\text { Relative range of } \\
\text { movement }\left({ }^{\circ}\right)\end{array}$ & $1.81 \pm 1.89$ & $4.51 \pm 3.52^{*}$ & \\
\hline
\end{tabular}

* Significantly different from normal sprinting $p \leq 0.05$

† Significantly different from waist attachment condition $\mathrm{p} \leq 0.05$ 
Table 4. Hip Joint kinematics (means and standard deviations) from the stance limb under the different conditions (normal, shoulder and waist).

\begin{tabular}{llll}
\hline $\begin{array}{l}\text { X (+=flexion/- } \\
\text { =extension) }\end{array}$ & Normal & Shoulder & Waist \\
& & & \\
\hline Angle at foot-strike $\left(^{\circ}\right)$ & $58.81 \pm 8.29$ & $67.08 \pm 8.18^{*}$ & $65.80 \pm 9.93^{*}$ \\
Angle at toe-off $\left({ }^{\circ}\right)$ & $-6.43 \pm 6.40$ & $-0.47 \pm 9.22^{*}$ & $0.36 \pm 8.33^{*}$ \\
$\begin{array}{l}\text { Peak flexion }\left({ }^{\circ}\right) \\
\text { Range of movement }\left({ }^{\circ}\right)\end{array}$ & $65.24 \pm 6.74$ & $67.08 \pm 8.18^{*}$ & $65.80 \pm 9.93^{*}$ \\
& & $67.55 \pm 8.84$ & $65.44 \pm 9.74$ \\
$\begin{array}{l}\text { Relative range of } \\
\text { movement }\left({ }^{\circ}\right)\end{array}$ & $65.24 \pm 6.74$ & $67.55 \pm 8.84$ & $65.44 \pm 9.74$ \\
\hline
\end{tabular}

* Significantly different from normal sprinting $p \leq 0.05$ 
Table 5. Knee joint kinematics (means and standard deviations) from the stance limb under the different conditions (normal, shoulder and waist).

\begin{tabular}{llll}
\hline X (+=flexion/-=extension) & Normal & Shoulder & Waist \\
\hline Angle at foot-strike $\left({ }^{\circ}\right)$ & $47.41 \pm 5.48$ & $54.28 \pm 6.60^{*}$ & $53.27 \pm 6.16^{*}$ \\
Angle at toe-off $\left({ }^{\circ}\right)$ & $15.76 \pm 5.79$ & $18.42 \pm 5.60^{*}$ & $18.95 \pm 5.87^{*}$ \\
Peak flexion $\left({ }^{\circ}\right)$ & $50.01 \pm 5.38$ & $56.62 \pm 5.49^{*+}$ & $54.81 \pm 5.68^{*}$ \\
Range of movement $\left({ }^{\circ}\right)$ & $31.65 \pm 6.57$ & $35.86 \pm 8.37^{*}$ & $34.33 \pm 8.12$ \\
Relative range of & $2.60 \pm 4.80$ & & $1.53 \pm 3.31$ \\
movement $\left({ }^{\circ}\right)$ & & & \\
& & & \\
\end{tabular}

* Significantly different from normal sprinting $p \leq 0.05$

† Significantly different from waist attachment condition $p \leq 0.05$ 
Table 6. Ankle Joint kinematics (means and standard deviations) from the stance limb under the different conditions (normal, shoulder and waist).

\begin{tabular}{|c|c|c|c|}
\hline $\begin{array}{l}\mathrm{X}(+=\text { dorsi-flexion/- } \\
=\text { plantar-flexion) }\end{array}$ & Normal & Shoulder & Waist \\
\hline Angle at foot-strike $\left({ }^{\circ}\right)$ & $2.72 \pm 5.89$ & $5.85 \pm 5.34^{*}$ & $4.76 \pm 6.69 *$ \\
\hline Angle at toe-off $\left({ }^{\circ}\right)$ & $-25.40 \pm 4.01$ & $-24.34 \pm 3.44$ & 3.05 \\
\hline Peak dorsi-flexion $\left({ }^{\circ}\right)$ & $24.32 \pm 4.82$ & $27.08 \pm 6.00 *$ & \\
\hline Range of movement $\left({ }^{\circ}\right)$ & $28.11 \pm 5.00$ & & \pm 5.22 \\
\hline Relative range of & $21.61 \pm 6.23$ & $21.22 \pm 5.93$ & $21.24 \pm 5.82$ \\
\hline
\end{tabular}

* Significantly different from normal sprinting $\mathrm{p} \leq 0.05$ 

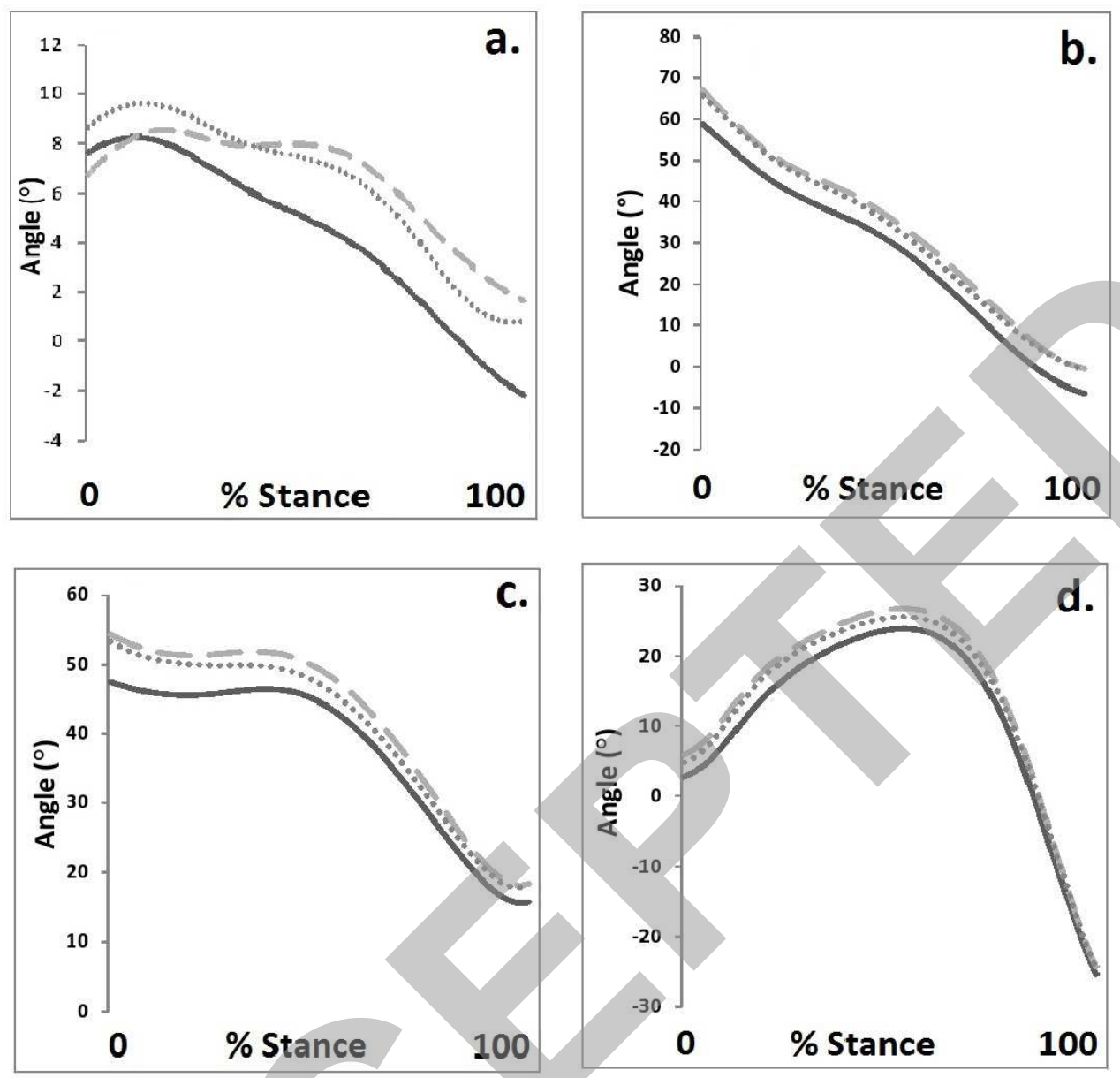

Copyright @ Lippincott Williams \& Wilkins. All rights reserved. 